\title{
Operative Therapy of Parkinson's Disease and the Role of Neurologist
}

\author{
Ellisa Dwijayanti ${ }^{1}$, Muhammad Hamdan ${ }^{1}$ \\ ${ }^{1}$ Department of Neurology, Airlangga University- Dr. Soetomo General Hospital, Surabaya, East Java, \\ Indonesia \\ Corresponding Author: Ellisa Dwijayanti
}

\begin{abstract}
Dopaminergic agents are still the mainstay of therapy in Parkinson's disease. Such dopamine replacement therapy results in a reduction in motor symptoms in the early stages of the disease. However, dopaminergic agents will cause motor (motor fluctuations and dyskinesia) and nonmotor (sensory, autonomic, and psychiatric) complications, $50 \%$ will occur after 5 years,

The operative approach has been shown to be effective in improving motor symptoms in PD. Deep brain stimulation (DBS), which is the second largest finding after levodopa, is still the first choice of operative treatment for PD, however, other operative therapies such as ablative therapy (radiofrequency, stereotactic radiosurgery, focused ultrasound thermal ablation, laser interstitial thermal therapy) have almost the same effectiveness. with DBS. As primary care physicians, it is very important for neurologists to understand the role in operative therapy that is currently developing.
\end{abstract}

Keywords: Parkinson's disease, surgery, neurologist, therapy.

\section{INTRODUCTION}

Parkinson's disease (PD) is a neurodegenerative disorder often affecting the elderly, characterized by generalized slowing of movement (bradykinesia) and at least one other symptom of tremor at rest (resting tremor) or rigidity (rigidity). (1) Another feature is loss of smell, sleep dysfunction, mood disorders, excessive salivation, constipation, and excessive body movements during sleep. (2) PD is caused by a decrease in the amount of dopamine in the brain that plays a role in controlling movement due to nerve cell damage in the substantia nigra pars compacta (SNc).(3)

PD is mostly experienced in the elderly and rarely under the age of 30 years. Usually begins at the age of 40-70 years and reaches a peak in the sixth decade. (4) PD affects one to two per 1000 people at any time, the prevalence increases with age, namely $1 \%$ of the population over 60 years of which $5 \%$ to $10 \%$ of patients have a genetic predisposition. (2) More in men with a male to female ratio of 3:2. (4)

Non-pharmacological aPDroach is an effective therapy to reduce motor symptoms of PD. Surgery and other advanced therapeutic options may be considered for patients whose symptoms cannot be adequately managed with medication alone. (6) Deep brain stimulation of the subthalamic nucleus (DBS-STN) can reduce the need for levodopa by $50-60 \%$ and time off can be reduced. by $61 \%$ and dyskinesia reduced to $59-75 \%$. DBS-STN is useful for the treatment of tremor, especially essential tremor.(1),(7)

\section{Deep Brain Stimulation (DBS)}

DBS is the implantation of electrodes into deep brain regions for the purpose of modulating nerve function to treat neurological/psychiatric conditions. The electrodes are implanted using a stereotactic technique and attached to an implantable pulse generator (IPG), which is 
usually placed subdermal under the clavicle.(8)

The DBS therapy system consists of a pair of electrodes implanted in the target brain tissue (thalamus, globus pallidus interna, or subthalamic nucleus). The electrode is connected to an implantable pulse generator (IPG), which is the source of energy in this DBS system, IPG is generally implanted in the subclavicular area in the space around the chest. the electrode and the IPG are connected by a connecting cable that is placed on the neck under the skin.(9)

DBS therapy produces monopolar or bipolar electrical stimulation of the targeted brain areas. The amplitude, frequency, and bandwidth of the stimulation can be adjusted to control symptoms. Patients can turn the stimulator on or off using the Access Review Therapy Controller or using a modified magnet. Stimulation parameters are with an amplitude of 1-3 V, a frequency of $135-185 \mathrm{~Hz}$, and a wavelength of $60-120$ msec.(9)

STN DBS can reduce the dose of antiparkinsonian dopaminergic drugs with improved motor function. Therefore, it is indicated for patients suffering from druginduced psychotic symptoms such as hallucinations and delusions. There is some evidence to suPDort this concept.(10),(11) Deuschl and colleagues conducted the first multicenter randomized clinical trial and reported a significant improvement in the untreated subset of UPDRS motor score III $(41 \%)$ with STN-DBS and a significant improvement. in quality of life $(24 \%)$ in patients with STN-DBS.(12)

\section{Ablative Surgery}

The goal of ablative therapy in neurological disorders is the selective destruction of targeted brain tissue volume. Several ablative procedures, based on various physical principles, have been used to date in the field of neurosurgery. This technique is used both to create therapeutic lesions in the brain to disrupt maladaptive brain tissue and to destroy abnormal tissue such as brain tumors.

\section{Thermal Radiofrequency Ablation}

The interstitial radiofrequency (RF) technique consists of creating the lesion using heat via intracranially placed electrodes coupled to an RF generator. These electrodes are electrically isolated except at the ends, where the active electrode is located. When the generator is activated, an electric current flows in the circuit between the active and dispersive electrodes. The electric field between the two contacts oscillates with the RF frequency and causes the nearby charge of ions in the electrolyte medium to move back and forth in space at the same high frequency, which is usually about 500,000 cycles per second for most modern generators. Frictional heating within the tissue resulting from RF ionic oscillations, i.e. current density is the basic mechanism by which the tissue heats up and by which RF heat lesions are created. The greatest heating occurs in the area with the highest current density, which is near the tip of the active electrode.(13)

Ventral intermediate nucleus (Vim) thalamotomy for tremor dominant PD shows 60 to $100 \%$ improvement using RF ablation, including a 57 to $90 \%$ long-term benefit over 2 to 15 years. Most adverse events for thalamotomy, including ataxia, dysarthria, and sensory/motor deficits, are associated with perilesional edema that resolves over time, usually over 1 week to 1 month, but varies depending on the size of the lesion. Studies involving unilateral pallidotomy with RF for PD have shown a median reduction of $30 \%$ in UPDRS III motor scores with improvement in tremor, bradykinesia, rigidity, gait, and balance. There was also improvement in dyskinesia of up to $90 \%$. Side effects of pallidotomy include: visual field deficits, paresis, and neuropsychological deficits, and are mostly transient because the duration of perilesional edema varies. Surgical risks 
associated with RF techniques also include bleeding and infection.(6)

\section{Laser interstitial thermal therapy (LITT)}

Lasers are a form of nonionizing radiation that produce a beam of coherent and collimating light energy. Their effect on tissues is due to two principles: absorption and scattering. Absorption is the conversion of laser energy into heat after the laser photons collide with molecules in the target tissue called chromophores (oxyhemoglobin and deoxyhemoglobin) are the main absorbers). This transfer of energy to the chromophore causes the release of heat and subsequent damage to the cell and adjacent structures. Scattering occurs when the trajectories of photons diverge by interacting with particles in the network, resulting in an increase in the spatial distribution of light and heat. After more than 50 years of technological development, lasers are used for stereotactic ablation in the form of magnetic resonance imaging (MRI) guided LITT.

\section{Radiosurgery}

Stereotactic radiosurgery (SRS) is an external ablative treatment modality that delivers a single large dose of radiation to a limited volume of intracranial targets, leaving the surrounding tissue intact. Computerized dose asymmetry planning and highly accurate radiation delivery systems are required to achieve this effect. The target location is determined by imageguided stereotaxy. In SRS energy is delivered to the target in the form of ionizing radiation, i.e. any radiation entity that has sufficient energy to remove electrons from atoms, thereby creating ions. In turn, these charged particles interact with living tissue in the target and produce biological responses such as cell death or mitotic termination. (14) Currently, several devices are used to perform SRS procedures, each based on a different source of ionizing radiation. For example, Gamma Knife Radiosurgery (GKRS), developed by Lars Leksell, utilizes gamma rays originating from the excitation of the 60Co nucleus. Linear accelerators are a more costeffective alternative to SRS and produce single beam high energy $\mathrm{X}$-rays that are focused by a special collimator with a view to narrowing, modulating or shaping the beam to a network volume.(13)

One study reported near-perfect improvement in tremor in about $88 \%$ of PD patients with SRS thalamotomy. SRS has also been shown to be equally effective and safe as RF lesions and DBS therapy for pallidal lesions.(6)

\section{Focused ultrasound thermal ablation}

MR-guided focused ultrasound (MRgFUS) is a new non-invasive technique used for thermal ablation. In this procedure, HIFU rays are targeted to the intracranial area using a hemispheric phased-array transducer that is attached to the skull. This allows the passage of ultrasonic waves through the maximum available skull area, thus avoiding overheating and brain damage. This device is also coupled with special software that allows correction of phase distortion and ultrasonic beam aberrations produced by skull irregularities. Ultrasonic mechanical energy is absorbed in the target volume of the focus and converted into heat which causes local tissue damage at the focus. At the same time, MRI and MR-thermography guidance allows accurate targeting and timing of energy deposition monitoring.(15)

Currently, MRgFUS is FDA approved for unilateral thalamotomy in tremor dominant ET and PD. Its use in pallidotomy and subthalamotomy is being investigated. In several studies, MRgFUS thalamotomy for tremor-dominant PD has shown an improvement from 30 to $60 \%$ in UPDRS III motor scores. Similar results have been reported with MRgFUS pallidotomy. In unblinded subthalamotomy MRgFUS, 9 patients who underwent a modified MRgFUS protocol showed an increase in UPDRS III motor score of $60.9 \%$ at 3 months. In another study targeting STN MRgFUS, 10 patients 
reported a 53\% improvement in UPDRS III motor scores within 6 months without significant side effects. In this study, there were a total of 38 adverse events during the 6 month follow-up.(16)

\section{Transplant and Gene Therapy}

Cell transplantation is considered a potential future treatment of PD. There have been trials using autologous and nonautologous cells. Human embryonic stem cells and induced pluripotent stem cells are some of the cells that have been included in this transplant study. One of the concerns with stem cell cell transplantation is the ethical limitations that must be considered.(17),(18) Since the first clinical trial in 1987 involving the transplantation of human fetuses rich in dopaminergic neurons of mesencephalic tissue into the striatum of PD patients, more research has been conducted. aimed to explore whether the grafted network of dopaminergic neurons would live and form connections in the brain, if the patient's brain could align and utilize the grafted neurons, and if the graft could result in significant clinical improvement. Clinical trials with cell therapy were intended to determine whether there is long-term improvement after restoration of striatal DA transmission by transplanted dopaminergic neurons. Experimental data from rodents and nonhuman primates show that fetal ventral mesencephalon intrastriatal grafted DA neurons exhibit many morphological and functional characteristics of normal DA neurons. Dopaminergic grafts can innervate the striatum in the brain, restore DA release that is regulated in the striatum, and can be functionally integrated into neural circuits.(16)

A new area being explored in PD treatment is gene therapy. Both non-disease and disease modifying transgenes are being investigated. Non-disease-modifying transgenes target GABA and dopamine synthesis pathways. Disease-modifying transgenes have the potential to slow disease progression. Both transgenes are too early in trials to be conclusive but offer promising results. Gene therapy addresses only motor symptoms, not nonmotor symptoms.(16)

The Role of the Neurologist in DBS: Preoperative, Intra-operative and Postoperative Evaluation

One of the main roles of a DBStrained neurologist is preoperative evaluation. Potential DBS candidates are carefully selected following a multidisciplinary and specialized evaluation to determine the risk-benefit ratio. DBS candidates should be considered resistant to trials of conventional treatment and, in some cases, behavior-based therapy. Patient expectations are carefully weighed in each case, and a determination is made as to whether DBS will potentially affect one or more disabling symptoms for the individual sufferer. This evaluation includes treatment optimization and behavioral treatment, as well as recording validated standard symptom rating scales. The current best practice for evaluation of DBS is evaluation by a multidisciplinary team, led by a DBStrained neurologist and neurosurgeon with expertise in stereotactic and functional neurosurgery, and including neurologists, psychiatrists, physical therapists, occupational therapists, speech therapists, and in some cases a social worker or financial advisor.

Each specialization will individually evaluate potential DBS candidates, and meet to discuss key questions (patient expectations, symptoms) targets, risk/benefit ratio, unilateral vs bilateral, staged vs single surgery, salvage clues for DBS failure, or hardware issues). Once the board's decision has been given, the patient is usually contacted to discuss details and, if aPDropriate, scheduled for surgery. A clear understanding of the required time commitment of the patient, family and medical team prior to surgery is paramount, as there will be many visits over the next 612 months.(19) Intra-operative includes understanding direct and indirect targeting 
and for DBS recording and microelectrode/macrostimulation.

The DBS neurologist plays an active role postoperatively by confirming the correct location of the lead, usually through imaging interpretation. They also recorded the thresholds for benefit and side effects at each point of contact on each DBS lead in order to confirm the imaging findings. The DBS neurologist must be skilled in identifying the best parameter settings (voltage, current, pulse width, frequency, scheduled stimulation, chronic stimulation). Patients are usually followed monthly for the first 6 to 12 months, and then periodically thereafter, for device checks. Important aspects for postoperative DBS treatment include drug management, end-oflife planning for battery life, troubleshooting device complications, and counselling / treatment for disease progression. (19)

\section{CONCLUSION}

Currently, many surgical therapies for PD have been developed, including DBS and ablative surgery. Both operative therapies have shown improvement in motor symptoms of PD as assessed by improvement in UPDRS III motor score. With the collaboration of pharmacological and non-pharmacological therapies, it is hoped that motor fluctuations and dyskinesia that arise due to the use of oral therapy can be reduced. As for other nonpharmacological therapies that are being developed, such as transplantation and gene therapy, which are currently still in the preclinical stage and information is still limited.

Neurologists play a very important role in the management of $\mathrm{PD}$, both in the management of pharmacological and nonpharmacological therapies. In the management of surgical therapy, a trained neurologist plays a role in preoperative, intraoperative and postoperative evaluation.

\section{Acknowledgement: None}

Conflict of Interest: None

\section{Source of Funding: None}

\section{REFERENCES}

1. Horstink M. Early (uncomplicated) Parkinson's disease and late (complicated) Parkinson's disease. In: European Handbook of Neurological Management. In Blackwell; 2006. p. 224-65.

2. Saman Zafar; Sridhara S. Yaddanapudi. Parkinson DIsease. Treasure Island (FL): StatPearls Publishing; 2021.

3. Kurniawan M, Suharjanti I PR. Panduan Praktik Klinis Neurologi. Jakarta: Perhimpunan Dokter Spesialis Saraf Indonesia; 2016.

4. Syamsudin T, Subagya AM. Buku Paduan Tatalaksana Penyakit Parkinson Dan Gangguan Gerak Lainya Kelompok Studi Movement Disorder PERDOSSI. 2015.

5. Setyono Erni; Gunarto, Untung JN. HUBUNGAN ANTARA MEROKOK DENGAN PENYAKIT PARKINSON DI RSUD PROF. DR. MARGONO SOEKARJO PURWOKERTO. MANDALA Heal [Internet]. 2010;(Vol 4, No 2 (2010): Mandala Of Health):81-6. Available from: http://jos.unsoed.ac.id/index.php/moh/article /view/755

6. Sharma VD, Patel M, Miocinovic S. Surgical Treatment of Parkinson's Disease: Devices and Lesion APDroaches. Neurotherapeutics. 2020;17(4):1525-38.

7. Dewanto G. Manajemen gejala motorik dan non-motorik pada Penyakit Parkinson. Neurona [Internet]. 2012;29 (3)(April):1530. Available from: http://www.neurona.web.id/paperdetail.do?id=812

8. Pycroft L, Stein J, Aziz T. Deep brain stimulation: An overview of history, methods, and future developments. Brain Neurosci Adv. 2018;2:239821281881601.

9. Weaver FM, Stern M, Harris C, Jr WJM, Reda D, Moy CS, et al. Bilateral Deep Brain Stimulation vs Best Medical Therapy for Patients With Advanced Parkinson's Disease. 2015;301(1):63-73.

10. Umemura A, Oka Y, Okita K, Matsukawa N, Yamada K. Subthalamic nucleus stimulation for Parkinson disease with severe medication-induced hallucinations or delusions. J Neurosurg. 2011;114(6):17015. 
Ellisa Dwijayanti et.al. Operative therapy of parkinson's disease and the role of neurologist.

11. Yoshida F, Miyagi Y, Kishimoto J, Morioka $\mathrm{T}$, Murakami N, Hashiguchi $\mathrm{K}$, et al. Subthalamic nucleus stimulation does not cause deterioration of preexisting hallucinations in Parkinson's disease patients. Stereotact Funct Neurosurg. 2009;87(1):45-9.

12. Deuschl G, Schade-Brittinger C, Krack P, Volkmann J, Schäfer H, Bötzel K, et al. A Randomized Trial of Deep-Brain Stimulation for Parkinson's Disease. N Engl J Med. 2006;355(9):896-908.

13. Franzini A, Moosa S, Servello D, Small I, DiMeco F, Xu Z, et al. Ablative brain surgery: an overview. Int $\mathrm{J}$ Hyperth [Internet]. 2019;36(2):64-80. Available from:

https://doi.org/10.1080/02656736.2019.161 6833

14. De Salles AAF, Gorgulho AA, Pereira JLB, McLaughlin N. Intracranial Stereotactic Radiosurgery. Concepts and Techniques. Neurosurg Clin N Am [Internet]. 2013;24(4):491-8. Available from: http://dx.doi.org/10.1016/j.nec.2013.07.001

15. Eames MDC, Farnum M, Khaled M, Jeff Elias W, Hananel A, Snell JW, et al. Head phantoms for transcranial focused ultrasound. Med Phys. 2015;42(4):1518-27.

16. Lee TK, Yankee EL. A review on Parkinson's disease treatment. Neuroimmunol Neuroinflammation [Internet]. 2021;8:[Online First]. Available from: http://dx.doi.org/10.20517/23478659.2020.58

17. Krauss JK, Lipsman N, Aziz T, Boutet A, Brown P, Chang JW, et al. Technology of deep brain stimulation: current status and future directions. Vol. 17, Nature Reviews Neurology. 2021. 75-87 p.

18. Yasuhara T, Kameda M, Sasaki T, Tajiri N, Date I. Cell Therapy for Parkinson's Disease. Cell Transplant. 2017;26(9):15519.

19. Hassan A, Okun MS. Emerging Subspecialties in Neurology: Deep brain stimulation and electrical neuro-network modulation. Neurology. 2013;80(5):47-50.

How to cite this article: Dwijayanti E, Hamdan M. Operative therapy of parkinson's disease and the role of neurologist. International Journal of Research and Review. 2021; 8(9): 43-48. DOI: https://doi.org/10.52403/ijrr.20210908 